\title{
Disturbances of the Natural Electric Field in the Atmosphere Before Technogenic Earthquakes
}

\author{
Dmitry Sirota ${ }^{1, *}$, and Vadim Ivanov ${ }^{1}$ \\ ${ }^{1}$ T.F. Gorbachev Kuzbass State Technical University, 650000, 28 Vesennyaya St., Kemerovo, Russia
}

\begin{abstract}
Modern seismology attaches great importance to the methods of short-term earthquake forecasting. One of these methods is a method based on the registration of disturbances of the atmospheric electric field and the field arising in rocks before earthquakes. Until now, this problem is discussed at the level of hypotheses. The mechanism of perturbations is unclear and the literature does not provide any convincing evidence of the explanation of the observed phenomena - flashes and columns of light, breakdown of electric cables in the earth a few hours before the earthquake and other phenomena. In the present article on the basis of the phenomenon of fast cracks loading discovered in the nineties of the last century [1] the explanation of these phenomena is offered by the solution of a direct problem about an electric field of the hearth of technogenic earthquake in the atmosphere.
\end{abstract}

\section{Introduction}

Over the past twenty years in the Kuzbass observed increase in seismic activity of the earth's crust, resulting in an increased number of dynamic manifestations of rock pressure in mines, and also did not appear earlier provoked by mining induced earthquakes of small force (mostly fifth to seventh energy class). Moreover, the depth of most of the foci of these earthquakes according to the Altai - Sayan geophysical service did not exceed one kilometer.

The modern point of view links the seismic event with the focal area for which the following definitions can be given

1) the focal area of the seismic event is a certain volume the area of rocks surrounding the place of the incipient rupture and corresponding to the area of irreversible deformations and maximum variations of mechanical stresses;

2) the effective focal area of the seismic event is the volume area of rocks including the focal area, and also a area of possible aftershocks.

These focal areas are associated with displacements on large tectonic faults in the earth's crust. From the very beginning, the process of their accumulation is scattered, but with the formation of larger cracks, the process of occurrence of microcracks is localized in a narrow area, which is a zone of growing trunk rupture. When the stress in the hearth reaches a critical value, the fault boards are formed, they slip relative to each other and a seismic event occurs. At the same time, the elastic energy stored in the surrounding rocks partially passes into heat, and partly into the energy of propagating seismic waves. It is along the faults that the relative

\footnotetext{
${ }^{*}$ Corresponding author: sdy.pm@kuzstu.ru
} 
displacements of rigid lithospheric plates often occur. In this case, the faults are divided into exploits when the maximum value of the tectonic component of the stresses is compressive; normal discharges, in the case where the maximum component of the tectonic stresses is tensile; and faults with displacement along the stretch (shifts).

Currently, there are many scientific articles devoted to the description of experiments on the relationship between the seismic event and harbingers of different nature: acoustic, electric, magnetic [2 - 13]. In [14] a large review of electromagnetic earthquake precursors is presented: local changes of electroconductivity of the earth, anomalies of telluric currents and geoelectric potentials, low-frequency variations of the earth's atmospheric electric field, low-frequency variations of the earth's magnetic field, low frequency electromagnetic noise in the ULF-band, radio emission and optical phenomena before earthquakes, release of radioactive gases from the earth's bowels, change in the content of subsurface hydrogen.

In the preparation of mining-tectonic shocks and man-made earthquakes, the focus of which is located at a relatively shallow depth, both on the earth's surface and in the atmosphere there are noticeable anomalies of the natural electric field, due to the charging state of cracks when they accumulate in the area of the hearth. In [1] was presented the mechanism, caused by the birth and rapid spread of microcracks where each microcrack in the process of propagation carries a charge of one sign in its vertex. This mechanism explains the long-term existence of the charging state in rocks due to the ability to continuously resume rapidly relaxing charges.

In this article we obtain estimates of the potential and intensity of a quasi-stationary electicity field generated by a three-dimensional object.

\section{Result and discussion}

\subsection{Potential and intensity of a field}

Let us assume that the containing space is the homogeneous isotropic triplex with planeparallel borders and the specific resistance $\rho_{1}, \rho_{2}, \rho_{3}$ and the field source is in the first layer. Let us first calculate the field of a point source (Fig. 1) in the coordinate system Oxyz, which is located in the 1 st layer.

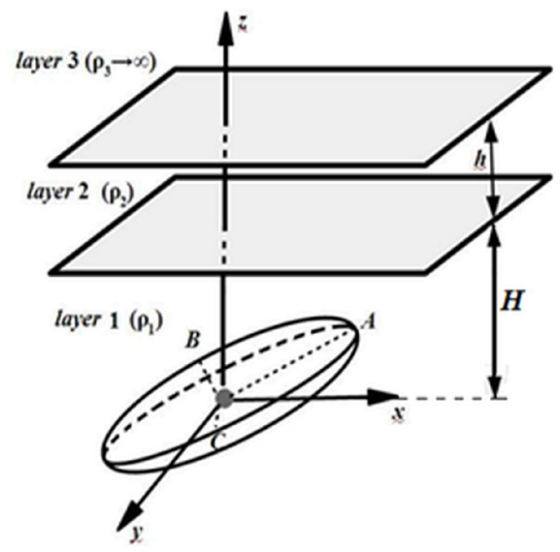

Fig. 1. To calculation of electromagnetic disturbance in the atmosphere from the focal area of mining - tectonic shocks and man-made earthquakes.

From geodynamics and seismology it is known [15] that earthquakes are associated with differences of the type of feat, discharge and displacement along the stretch. The formation 
of large faults in the earth's crust is due to the multiple accumulation of microcracks in the focal zone, their association and the formation of large cracks (forks), the connection of which occurs by breaking the bridges between them (the earthquake itself), as well as with a discontinuous displacement of the rupture sides, called intermittent sliding [15].

As well as known, the solution of this problem for the potential of a point source in the atmosphere (3rd layer) looks like:

$$
u_{3}=\frac{\rho_{2} I}{2 \pi} \int_{0}^{\infty} \frac{\left(1-K_{1}\right) e^{-m z} J_{0}(m r)}{1+K_{1} e^{-2 m h}} d m
$$

where $\rho_{2}$ is the specific resistivity of the second layer $\left(\Omega^{*} \mathrm{~m}\right) ; K_{1}=\frac{\rho_{2}-\rho_{1}}{\rho_{2}+\rho_{1}}$ is the reflection coefficient of the second layer; $J_{0}(m r)$ is the Bessel function of zero order; $I$ is the point source current; $r=\sqrt{x^{2}+y^{2}}$ is the polar radius; $h$ is the thickness of the second layer.

In [16 - 18] we calculated the potential for a flat shape and a cylinder. In this paper we will consider the general case of a three-dimensional body and ellipsoid for example.

Denoting through $j_{3 D}$ the volume density of currents in the source of destruction and integrating (1) the volume of an ellipsoid with semiaxis $A, B, C$, we obtain the following expression for the potential of the focal zone in the atmosphere:

$$
U_{3}=\frac{\rho_{2} j_{3 D}}{2 \pi} \iiint_{V_{p}}\left[\int_{0}^{\infty} \frac{\left(1-K_{1}\right) e^{-m z} J_{0}(m r)}{1+K_{1} e^{-2 m h}} d m\right] d V_{p}
$$

where $r=\sqrt{\left(x_{M}-x_{p}\right)^{2}+\left(y_{M}-y_{p}\right)^{2}}, \quad z=z_{M}-z_{p}, \quad V_{p} \quad-$ three-dimensional body (ellipsoid Fig . 1); $x_{p}, y_{p}, z_{p}$ - the coordinates of the point $P$ inside the focal zone, which in (2) is the external integration; $x_{M}, y_{M}, z_{M}$ - the coordinates of the observation point in the atmosphere).

Let us transform the expression in (2) integrals to a dimensionless form by notations $w / h=\bar{w}$, where the dimensions of a quantity of $w$ is the meter. After such change the elementary volume $d V_{p}$ is converted to the following dimensionless quantity $d V_{p}=A^{3} d \bar{V}_{p}$ and the remaining quantities will be converted with the changes $m h=p$ and $d m=d p / h$. Here and then for simplicity we will not use line over variables. The expression (2) can be represented as

$$
U_{3}=\frac{\rho_{2} h^{2} j_{3 D}}{2 \pi} \iiint_{V_{p}}\left[\int_{0}^{\infty} \frac{\left(1-K_{1}\right) e^{-p z} J_{0}(p r)}{1+K_{1} e^{-2 p}} d p\right] d V_{p}
$$

where $r=\sqrt{\left(x_{M}-x_{p}\right)^{2}+\left(y_{M}-y_{p}\right)}, z=z_{M}-z_{p}$.

To calculate the inner improper integral let us turn to the Taylor series as follows. Since $\left|K_{1}\right|<1$, the expression $\frac{e^{-p\left(z_{M}-z_{p}\right)}}{1+K_{1} e^{-2 p}}$ can be expanded in a series 


$$
\begin{aligned}
& \sum_{n=0}^{\infty}\left(-K_{1}\right)^{n} e^{-p\left(2 n+z_{M}-z_{p}\right)} \text {. Then, using well known Weber's } \\
& \int_{0}^{\infty} J_{0}(m r) e^{-m z} d m=\frac{1}{\sqrt{r^{2}+z^{2}}} \text {, an improper integral in (3) can be represented as: } \\
& \int_{0}^{\infty} \frac{\left(1-K_{1}\right) e^{-p\left(z_{M}-z_{p}\right)} J_{0}(p r)}{1+K_{1} e^{-2 p}} d p=\left(1-K_{1}\right) \sum_{n=0}^{\infty} \frac{\left(-K_{1}\right)^{n}}{\sqrt{r^{2}+\left(2 n+z_{M}-z_{p}\right)^{2}}},
\end{aligned}
$$

Substituting (4) in (3) and changing the order of summation and integration, we obtain:

$$
U_{3}=\frac{\rho_{2} h^{2} j_{3 D}}{2 \pi}\left(1-K_{1}\right) \sum_{n=0}^{\infty}\left(-K_{1}\right)^{n} \iiint_{V_{p}} \frac{d V_{p}}{\sqrt{r^{2}+\left(2 n+z_{M}-z_{p}\right)^{2}}},
$$

To calculate the three dimensional integral, we define the boundary of the region as a function $R(\varphi, \psi)$ in the spherical coordinate system as follows: $x_{p}=\rho \sin \psi \cos \varphi$, $y_{p}=\rho \sin \psi \sin \varphi, z_{p}=\rho \cos \psi, 0 \leq \varphi \leq 2 \pi, 0 \leq \psi \leq \pi$ and $d V_{p}=\rho^{2} \sin \psi d \varphi d \psi d \rho$.

Bellow we will modeling the focal area of earthquakes as ellipsoid with the axes $A>B>C$. The dimensionless equation of the inclined ellipsoid in spherical coordinate system will look like

$$
R(\varphi, \psi)=\frac{A B C}{h \sqrt{T_{1}^{2}+T_{2}^{2}+T_{3}^{2}}},
$$

where

$$
T_{1}=(\sin \psi \cos \varphi \cos \gamma-\cos \psi \sin \gamma) B C,
$$

$T_{2}=\sin \psi \sin \varphi A C$, $T_{3}=(\sin \psi \cos \varphi \sin \gamma+\cos \psi \cos \gamma) A B, \gamma$ is the angle from intervals $23^{0}<\gamma<45^{0}$ or $45^{0}<\gamma<65^{0}$.

Thus we can write the $3 \mathrm{~d}$ integral from (5) as

$$
\iiint_{V_{p}} \frac{d V_{p}}{\sqrt{r^{2}+\left(2 n+z_{M}-z_{p}\right)^{2}}}=\int_{0}^{2 \pi} d \varphi \int_{0}^{\pi} \sin \psi d \psi \int_{0}^{R(\varphi, \psi)} \frac{\rho^{2} d \rho}{\sqrt{\rho^{2}+b \rho+a}}
$$

where $a=x_{M}^{2}+y_{M}^{2}+\left(2 n+z_{M}\right)^{2}, b=-2\left[x_{M} \sin \psi \cos \varphi+y_{M} \sin \psi \sin \varphi+\left(2 n+z_{M}\right) \cos \psi\right]$

The inner integral over variable $\rho$ we can calculate as

$$
\int_{0}^{R(\varphi, \psi)} \frac{d \rho}{\sqrt{\rho^{2}+b \rho+a}}=\left\{\begin{array}{c}
\ln \left|\frac{\sqrt{R^{2}(\varphi, \psi)+b R(\varphi, \psi)+a}+R(\varphi, \psi)+0,5 b \mid, \quad \text { if } b^{2}-a \neq 0}{0,5 b+\sqrt{a}}\right| \begin{array}{c}
\sqrt{a} \\
\end{array} \\
\ln \mid \frac{R(\varphi, \psi)-\sqrt{a} \mid, \quad \text { if } \quad b=2 \sqrt{a}}{\ln \left|\frac{R(\varphi, \psi)}{\sqrt{a}}-1\right|, \quad \text { if } \quad b=-2 \sqrt{a}}
\end{array} .\right.
$$


To calculate the intensity of a field we can use the difference schemes of 2 nd order of accuracy:

$$
E_{x}=\frac{U(x+\Delta)-U(x-\Delta)}{2 \Delta}, E_{y}=\frac{U(y+\Delta)-U(y-\Delta)}{2 \Delta},
$$

Let us calculate dimensionless potential and intensity for the ellipsoid as the field source with parameters $A=50, B=25, C=10, h=50, H=100 \mathrm{~m}, \gamma=30^{0}, 45^{\circ}, 60^{\circ}$, $K_{1}=-0,6$ and measurement point with coordinates $y=0, x \in[-600 ; 600] \mathrm{m}$. Let us to make a calculation in free mathematical tool Scilab. The graphical results of it are in Fig 2.

\subsection{Calculation the volume density of currents}

The volume density of currents in the focal zone can be found in accordance with [1] according to the formula

$$
j_{3 D}=\frac{2 \pi}{\varepsilon_{0} \varepsilon_{1} \cdot \rho_{1}} L Q N^{\prime} t_{p}
$$

where $L$ - the linear size of the formed microcracks, $(\mathrm{m}) ; N^{\prime}-$ the rate of microcrack formation, $\left(\mathrm{m}^{-3} \mathrm{~s}^{-1}\right) ; t_{p}$ - the time of relaxation of charges at the top of the crack, (s.); $Q_{-}$ the linear charge density per unit length of the crack front, $(\mathrm{C} / \mathrm{m}) ; \varepsilon_{0} \varepsilon_{1}-$ the absolute dielectric permeability of rocks in layer $1,(\mathrm{~F} / \mathrm{m}) ; \rho_{1}$ - specific electrical resistivity of rocks 1 layer $(\mathrm{Ohm} \cdot \mathrm{m})$.

Let us do the dimensional analysis of (9):

$$
\left[j_{3 D}\right]=\frac{[\mathrm{m}] \cdot[\mathrm{C} / \mathrm{m}] \cdot\left[\mathrm{m}^{-3} \cdot \mathrm{s}^{-1}\right] \cdot[\mathrm{s}]}{[\mathrm{F} / \mathrm{m}] \cdot[\mathrm{Ohm} \cdot \mathrm{m}]}=\frac{[\mathrm{C}]}{[\mathrm{F}] \cdot\left[\mathrm{Ohm} \cdot \mathrm{m}^{3}\right]}=\frac{[\mathrm{C}]}{\left[\mathrm{C} \cdot \mathrm{V}^{-1}\right] \cdot\left[\mathrm{V} \cdot \mathrm{A}^{-1} \cdot \mathrm{m}^{3}\right]}=\frac{[\mathrm{A}]}{\left[\mathrm{m}^{3}\right]} \text {. }
$$

The number of microcracks accumulated by the time of the earthquake in the volume unit can be determined from the concentration destruction criterion [1]: $N^{-\frac{1}{3}} \approx 3 L$ and hence $N \approx 0,037 L^{-3}$.

The average rate of micro-cracking at the first longest stage of earthquake preparation can be determined by the formula: $N^{\prime}=N / t$, where $t$ is the time of the formation of the hearth of destruction in seconds (the time between two subsequent events of one energy class) .

We can calculate the time in two ways: following M. A. Sadovsky-K. Tsuboi [19] and following S.N.Zhurkov [20].

Let us consider the first way. The time value which is determined from the seismic law of repeatability of M. A. Sadovsky-K. Tsuboi [18] as:

$$
\lg t=\frac{1}{3} \lg W-3,5,
$$

where $t$ - time of formation of the hearth of destruction in years, $W$ - the allocated seismic energy, J.

Thus, for the average rate of microcrack formation we obtain an estimate:

$$
N^{\prime} \approx 3,761 \cdot 10^{-6} W^{-\frac{1}{3}} L^{-3}
$$


According to the empirical laws of seismology, the size of the earthquake source is associated with the energy released as $A \approx 0,2 \cdot W^{\frac{1}{3}}$.
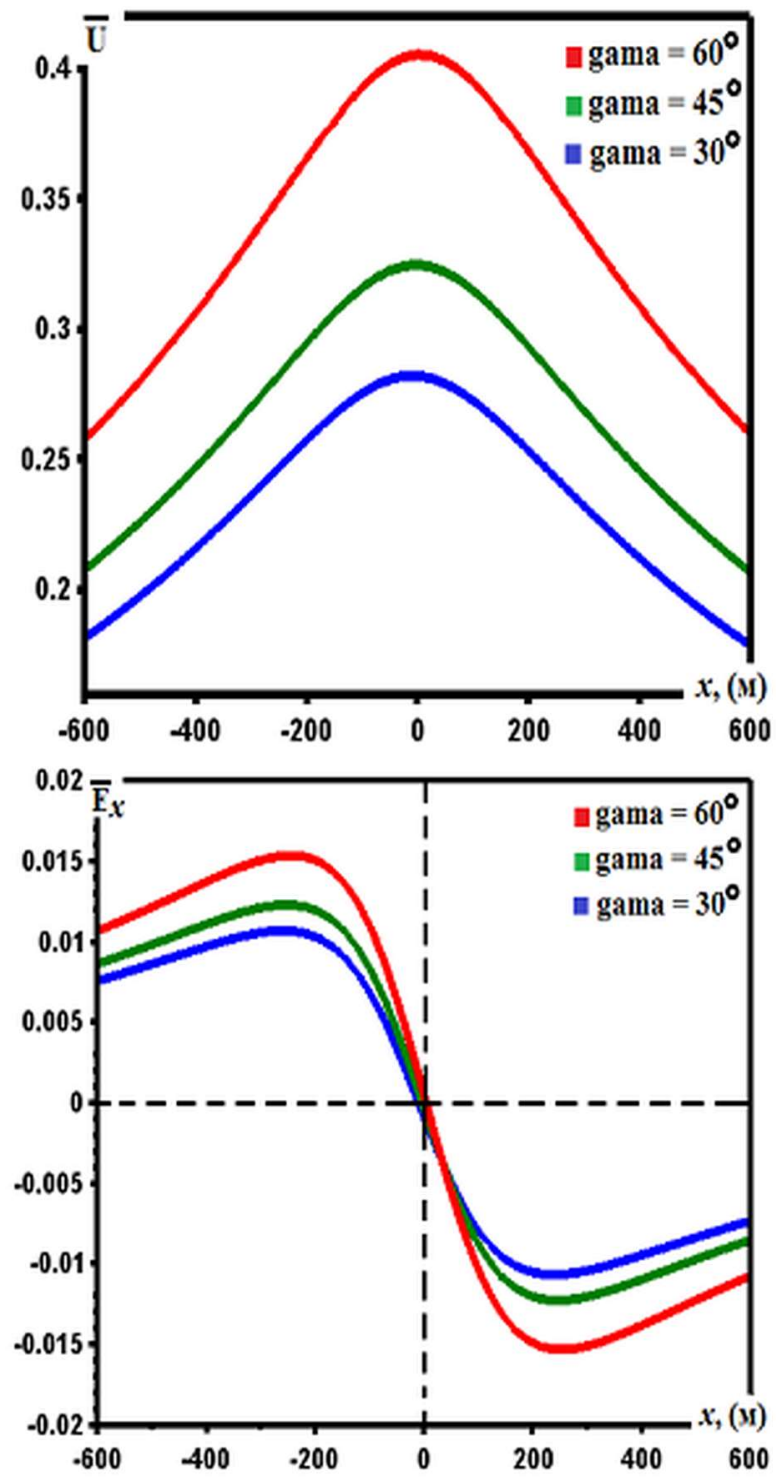

Fig. 2 The values of the dimensionalless potential and the intensity of field.

Using (10 - 11) and typical values for granodiorite, sandstone, quartz diorit $L=10^{-4} \mathrm{~m}$, $Q=6 \cdot 10^{-11} \mathrm{C} / \mathrm{m}, \quad t_{p}=10^{-5} \mathrm{~s}, \quad \varepsilon_{0} \varepsilon_{1}=8,854 \cdot 10^{-12} \mathrm{~F} / \mathrm{m}$ rewrite the (9) as $j_{3 D}=\frac{3,20277 \cdot 10^{-2}}{\rho_{1} A}$. Hence if $\rho_{2}=100, \rho_{1}=10^{4} \mathrm{Ohm} \cdot \mathrm{m}, h=50, A=100 \mathrm{~m}$ the 
dimensional ratio for potential is $K_{U}=\frac{\rho_{2} h^{2} j_{3 D}\left(1-K_{1}\right)}{2 \pi}=5,047 \cdot 10^{-3} \mathrm{~V}$ and for intensity is $K_{E}=\frac{K_{U}}{h}=1,009 \cdot 10^{-5} \mathrm{~V} / \mathrm{m}$.

Let us consider the second way. The lifetime $t$ is determined from the kinetic concept of strength by S.N. Zhurkov:

$$
t=t_{0} \exp \left[\frac{U_{0}-\Omega \sigma_{p}}{k T}\right],
$$

where $t_{0}$ is the period of atomic thermal vibrations, s.; $\Omega$ is the activation volume, $\mathrm{m}^{3} ; U_{0}$ is the activation energy of fracture, $\mathrm{J} ; k$ is the Boltzmann's constant, $\mathrm{J} / \mathrm{K}^{0} ; T$ is the absolute probability the rock temperature, $\mathrm{K}^{0} ; \sigma_{p}$-stress, removed by the quakes, $\mathrm{Pa}$.

Using typical values for granodiorite, sandstone, quartz diorite $U_{0}=1,5 \cdot 10^{-19} \mathrm{~J}$, $\Omega=3,87 \cdot 10^{-27} \mathrm{~m}^{3}$ and $T=293 \mathrm{~K}^{0}, \sigma_{p}=4,5 \cdot 10^{4} \mathrm{~N} / \mathrm{m}^{2}, t_{0}=10^{-13} \mathrm{~s}$ and $L=10^{-4} \mathrm{~m}$, $Q=6 \cdot 10^{-11} \mathrm{C} / \mathrm{m}, t_{p}=10^{-5} \mathrm{~s}, \varepsilon_{0} \varepsilon_{1}=8,854 \cdot 10^{-12} \mathrm{~F} / \mathrm{m}$ rewrite the (9) as $j_{3 D}=\frac{1,45671}{\rho_{1}}$.

Hence If $\rho_{2}=100, \rho_{1}=10^{4} \mathrm{Ohm} \cdot \mathrm{m}, h=50 \mathrm{~m}$, then the dimensional ratio for potential is $K_{U}=\frac{\rho_{2} h^{2} j_{3 D}\left(1-K_{1}\right)}{2 \pi}=11,4774 \mathrm{~V}$ and for intensity is $K_{E}=\frac{K_{U}}{h}=0,2295 \mathrm{~V} / \mathrm{m}$. If we change the value $\sigma_{p}$ from $\sigma_{p}=4,5 \cdot 10^{4} \mathrm{~N} / \mathrm{m}^{2}$ to $\sigma_{p}=4,5 \cdot 10^{6} \mathrm{~N} / \mathrm{m}^{2}$ then dimensional ratio will transform to $K_{U}=796,841 \mathrm{~V}$ and for intensity is $K_{E}=15,937 \mathrm{~V} / \mathrm{m}$.

\section{Conclusion}

Thus, at the last stage of preparation of a large man-made earthquakes, the electric field intensity in the atmosphere can reach quite high values-from $100-300 \mathrm{mV} / \mathrm{m}$ which can be registered by rather simple measuring instruments such as conventional electrometers and serve as a short-term harbinger of an earthquake.

\section{References}

1. V. V. Ivanov Physical bases of electromagnetic processes at formation of the hearth of destruction in an array of rocks (KuzSTU, Kemerovo, 1994)

2. V. A. Mullayarov, V. V. Argunov, L. M. Abzaletdinova, and V. I. Kozlov, Nat. Hazards Earth Syst. Sci., 12, 3181 (2012)

3. H. G. Silva, M. Bezzeghoud, J. P. Rocha and all., Nat. Hazards Earth Syst. Sci., 11, 241 (2011)

4. M. Hayakawa, Nat. Hazards Earth Syst. Sci., 11, 301 (2011)

5. A. Ramirez-Rojas, L. Telesca, F. Angulo-Brown, Nat. Hazards Earth Syst. Sci., 11, 219 (2011)

6. H. G. Silva, M. Bezzeghoud, A. H. Reis at all., Nat. Hazards Earth Syst. Sci., 11, 987 (2011)

7. P. F. Biagi, R. Piccolo, V. Capozzi, Natural Hazards and Earth System Sciences 3: 197 (2003) 
8. A. Konstantaras, M. R. Varley, Natural Hazards and Earth System Sciences 4: 641 (2004)

9. P. F. Biagi, L. Castellana, T. Maggipinto Nat. Hazards Earth Syst. Sci., 7, 423 (2007)

10. F. Bella, R. Bella, P. Biagi at all., Annali di geofisica, XXXVII(5), 1131 (1994)

11. Yu-Lin Zhao, Bi-Ru Zhao, Fe-Ye Qian, Annali di geofisica, XL(2), 241 (1997)

12. G. Vargemezis, J. Zlotnicki, G.N. Tsokas, Annali di geofisica 44(2), 205 (2001)

13. 13.R. Teisseyre, Annali di geofisica, XL(2), 297 - 305, (1997)

14. V. V. Surkov The electromagnetic effect in earthquakes and burst (MMI, Moscow, 2000)

15. D. G. S. Tercott Geodynamics. Geological applications of solid state physics (MMI, Moscow, 1985)

16. D. Sirota, V. Ivanov, E3S Web of Conferences, 21, 01017, 1 (2017)

17. D. Sirota, V. Ivanov, V. Khyamyalyaynen E3S Web of Conferences, 15, 01012, 1 (2017)

18. D. Yu. Sirota, V. V. Ivanov Russian Geology and Geophysics, 59, 105 (2018)

19. S. N. Zhurkov, Int. J. Fract. Mech. 1(4), 311 (1964)

20. V.I. Myachkin Processes of preparation of earthquakes (MMI, Moscow, 1978). 